\title{
HLA-A*0201-Restricted VEGFR1 Peptide Vaccine
}

National Cancer Institute

\section{Source}

National Cancer Institute. HLA-A*0201-Restricted VEGFR1 Peptide Vaccine. NCI

Thesaurus. Code C77878.

A cancer vaccine containing an HLA-A*0201-restricted vascular endothelial growth factor receptor 1 (VEGFR1) peptide (sequence: TLFWLLLTL) with potential immunostimulatory and antitumor activities. Upon administration, HLA-A*0201-restricted VEGFR1-derived peptide vaccine may stimulate a cytotoxic $T$ lymphocyte $(C T L)$ response against tumor cells expressing VEGFR1, resulting in tumor cell lysis and decreased tumor growth. HLAA*0201 is an MHC class I molecule that presents antigenic peptides to CD8+ T cells; epitope design restricted to epitopes that bind most efficiently to HLA-A*0201 may improve antigenic peptide immunogenicity. 